Verunreinigungen des sogenannten chemisch reinen Glycerins.

I. Bronze der Reiterstatue Louis XIV., von Keller 1699 gegossen; II. Statue Henri IV.; III. Reiterstatue Ludwig XIV., von Goo; IV. Bronze zur Minervastatue, Paris; V. Bronze zur Napoleonsstatue, sehr gelb; VI. englisches Gussmessing, durch schöne Farbe und Schärfe des Gusses ausgezeichnet.

(1-V, Graham-Ot to's Lehrbuch der Chemie, Bd. II, 2. Abth. S. 830 , Aufl. II; VI, Jahresbericht der Chemie von Liebig und Kopp 1853, S. 726.).

Die Mischungen variiren sehr bedeutend, sicher auch an Farbe, Härte u. dgl. Die gefundene Zusammensetzung der Legirung Peter Vischer's ergiebt unbedeutende Beimengungen von Eisen, Mangan, Cadmium und Nickel, welche jedenfalls als Verunreinigungen aufzufassen sind; die Menge von Blei, wenig mehr als 1 Procent, ist jedoch vielleicht als wesentlich zu betrachten, analog dem Gehalte der anderen Gemische. Die unter VI aufgeführte engliche Legirung zeichnet sich durch einfache Zusammensetzung aus.

\title{
Ueber Verunreinigungen des sogenannten chemisch reinen Glycerins.
}

\author{
Von C. Schepky in Breslau.
}

Da die medicinische Wirkung des Glycerins wesentlich von dessen Reinheit abhängt, und gewisse Verunreinigungen statt der gewiunschten Wirkung oft deren Gegentheil hervorrufen, untersuchte $\mathrm{Hager}$ (Hager's pharmac. Centralhalle, 1867, S. 18.) Glycerin, welches statt der milden Eigenschaften des chemisch reinen entschieden erhitzende zeigte, und fand darin Oxalsäure neben Ameisensäure, in anderen Sorten Glycerins fand er Ammoniak.

Ausser den von $\mathrm{H}$ a ger angeführten Verunreinigungen habe ich in dem als purum und purissimum in den Handel kommenden Glycerin noch folgende beobachtet: 
Verunreinigungen des sogenannten chemisch reinen Glycerins. 17

1) Salpetersäure; leicht nachweisbar durch Eisenoxydulsalz und Schwefelsäure; sie kommt in allen Glycerinen vor, aus denen man das Chlor durch salpetersaures Silberoxyd entfernt hat.

2) Flüchtige Fettsäuren: Auf Zusatz einer kleinen Menge Salz - oder Salpetersäure, an dem bekannten ranzigen Geruch zu erkennen. Sie stammen aus dem Rohmaterial.

3) Alkalien. Man verdampft bis zur Trockne, resp. äschert ein, und prüft den Rückstand nach einer bekannten Methode. -- Die Alkalien finden sich meist in den Glycerinen, welche fliuchtige Fettsäuren enthalten und werden wohl vom Fabrikanten zugesetzt, um den Geruch nach diesen Säuren wegzuschaffen.

Kleine Mengen, sesp. Spuren von Chlor und Kalk, so wie von Schwefelsäure kommen zuweilen auch in den sogenannten reinen Glycerinen, welche nicht destillirt sind, vor; wo dies der Fall ist, war die Reinigung des Rohmaterials eine mangelhafte.

Was die von Hager angeführte Verunreinigung mit Oxalsäure anbelangt, so dürfte dieselbe wohl daher rühren, dass der betreffende Fabrikant das Rohglycerin durch oxalsaure Salze von seinem Kalkgehalt befreite.

Im Allgemeinen sind überhaupt nur die Glycerine als chemisch rein zu betrachten, welche durch Destillation gereinigt sind; alle anderen, welche nur chemisch gereinigt sind, enthalten stets Verunreinigungen, da alle bisherigen chemischen Raffinirungen stets nur eine auffallende Verunreinigung durch eine minder auffallende ersetzen. . (Breslauer Gewerbe-Blatt).

In den von mir untersuchten Glycerin-Proben habe ich Spuren von Kalk (im Rückstande von der Einäscherung) und Salpetersäure gefunden. Die Gegenprobe mit concentrirter Schwefelsäure ergab eine leichte Bräunung, nicht aber die braune Zone, diese entstand auch nicht sofort, sondern erst nach längerer Berührung der Flüssigkeitsschichten.

\section{Hirschberg.}

